\title{
Generation of baryon inhomogeneities in the electroweak phase transition
}

\author{
Ariel Mégevand ${ }^{1}$ and Francisco Astorga ${ }^{2}$ \\ 1 IFAE, Universitat Autònoma de Barcelona, \\ 08193 Bellaterra (Barcelona), Spain and \\ 2 Instituto de Física y Matemáticas, Universidad Michoacana de San Nicolás de Hidalgo, \\ Edificio C-3 Cd. Universitaria, A. Postal 2-82, 58040 Morelia, Michoacán, Mexico
}

(Dated: November 1, 2018)

\begin{abstract}
It is known that baryon number inhomogeneities may arise as a consequence of electroweak baryogenesis. Their geometry, size, and amplitude depend on the parameters that characterize the baryogenesis mechanism, as well as on those that determine the phase transition dynamics. We investigate this parametric dependance. We show that in the case of the minimal supersymmetric standard model, the geometry of the inhomogeneities most probably consists of spherical regions of high density surrounded by low-density walls, in contrast to the case of the minimal standard model. In this supersymmetric extension we find that density contrasts of up to a factor of 100 may arise. This amplitude increases for higher values of the latent heat or lower values of the bubble wall tension, and can be significantly larger in different extensions of the standard model. Such inhomogeneities may thus affect the dynamics of the subsequent quark-hadron phase transition.
\end{abstract}

\section{INTRODUCTION}

The generation of inhomogeneities in the baryon number density is a possible outcome of the QCD and the electroweak phase transitions [1, 2, 3, 4]. These may arise as a consequence of the perturbations produced by the walls of expanding bubbles in the surrounding plasma. In the QCD case, baryons, which are much heavier in the hadron phase than in the deconfined quark phase, are pushed away by the walls of expanding bubbles as the phase transition develops. Then, as the volume occupied by the hadron phase grows at the expense of the quark phase, baryons are driven into small regions of space [1]. Hence, it is believed that the general geometry of the QCD inhomogeneities is that of localized clumps of high density surrounded by voids of low density.

In the electroweak phase transition, baryon number inhomogeneities can arise as a consequence of the generation of the baryon asymmetry of the Universe (BAU), whether the latter is produced through the standard mechanism of electroweak baryogenesis [3] or in electroweak cosmic strings 4]. In general, the electroweak phase transition provides the three Sakharov conditions [5] for baryogenesis, namely, baryon number violation, $C$ and $C P$ violation, and a departure from thermal equilibrium. These conditions must combine in such a way as to produce a net difference between number densities of baryons and antibaryons. Interestingly, the idea of electroweak baryogenesis requires physics beyond the minimal standard model (SM) in order to give a quantitatively successful result. The basic idea is the following (for reviews on electroweak baryogenesis see [6]).

The standard mechanism assumes a first-order phase transition in order to achieve the 
non-equilibrium condition. As bubbles expand, a chiral flux is injected in front of their walls due to $C P$ violating interactions of the bubble walls with the particles of the plasma. Thus, an asymmetry between left handed quarks and their antiparticles is generated near the interfaces. This asymmetry biases the baryon number violating sphaleron processes in the symmetric phase. The resulting baryon asymmetry is caught by the walls and enter the bubbles. In order to avoid the washout of the generated BAU when equilibrium is restored, the sphaleron processes must be suppressed in the broken symmetry phase. This requirement imposes a condition on the value of the Higgs field $\phi$ inside the bubbles [7],

$$
\phi_{m}(T) / T \gtrsim 1 .
$$

Here, $\phi_{m}$ is the vacuum expectation value (VEV) of $\phi$ at temperature $T$, which corresponds to the global minimum of the free energy. It plays the role of an order parameter, and the condition (11) states that the phase transition must be strongly first-order. This usually constrains the parameters of the theory.

The generated BAU has a strong dependence on the velocity $v_{w}$ of bubble walls. On one hand, if the velocity is too large, the left-handed density perturbation will pass so quickly through a given point in space that sphaleron processes will not have enough time to produce baryons. Thus, the resulting baryon number density $n_{B}$ will be small in this case. On the other hand, for very small velocities thermal equilibrium will be restored and the baryon asymmetry will be erased by sphalerons. Hence, the baryon production will be small again. Therefore, the generated baryon number has a maximum for a certain wall velocity $v_{w}=v_{\text {peak }}$, which can be estimated by comparing the baryon number violation time scale with the time of passage of the chiral asymmetry [3, 8, 9]. Such estimates give a small value $v_{\text {peak }} \sim 10^{-2}$, which is confirmed by numerical calculations [10, 11].

This dependance of $n_{B}$ on the bubble wall velocity is important for electroweak baryogenesis, since it establishes a preference for models in which the velocity is close to $v_{\text {peak }}$. On the other hand, the velocity of bubble expansion is not constant throughout the phase transition [3, 12, 13, 14]. The variation of $v_{w}$ causes a variation of the local baryon density left behind by the walls along the bubble radius. It is thus evident that generating the observed BAU in the electroweak phase transition entails the formation of inhomogeneities in the baryon number density. These inhomogeneities are spherically symmetric and centered at the nucleation points.

A general feature of first-order phase transitions is the slow-down of bubble expansion due to the release of latent heat [13]. In the case of the electroweak phase transition, it is known that the velocity of bubble walls may decrease from an initial value $v_{i} \sim 10^{-1}-10^{-2}$ to a minimum velocity $v_{m} \sim 10^{-3}-10^{-4}$, but the variation can be larger, depending on the model [3, 14]. The maximum variation of the baryon density $n_{B}\left(v_{w}\right)$ as $v_{w}$ decreases from $v_{i}$ to $v_{m}$ determines the amplitude of the baryon inhomogeneities generated inside each bubble. The exact profile $n_{B}(r)$ along the radial direction and the general geometry of the inhomogeneities depend on the evolution of $v_{w}(t)$. For instance, if the initial velocity $v_{i}$ is close to the peak velocity $v_{\text {peak }}$, then clumps of high baryon density will be formed, surrounded by voids of low density. On the contrary, if $v_{m} \simeq v_{\text {peak }}$, walls of high density will surround voids of low density. The size of the inhomogeneities is roughly given by the mean separation between the centers of bubbles at the end of the phase transition.

The survival of the inhomogeneities at later epochs depends on the amplitude of the fluctuations as well as on the separation of the centers of fluctuations, which must be larger than the diffusion length of baryons for that fluctuation amplitude. In general, the inhomogeneities will have different cosmological consequences, depending on the epoch in which 
the phase transition occurs. Baryon inhomogeneities generated in the QCD phase transition have been intensively investigated due to their influence on Big Bang Nucleosynthesis (BBN) [1, 2, 15]. On the contrary, baryon inhomogeneities generated at the electroweak scale are not expected to survive long enough to affect BBN [3, 4].

However, electroweak inhomogeneities may survive until the QCD scale [16, 17]. Then, the dynamics of the quark-hadron phase transition may be affected by their presence 16, 18, 19]. This is because the critical temperature $T_{c}$ of the quark-hadron phase transition depends on the chemical potential, and therefore on the number density, being $T_{c}$ lower in regions with higher baryon density [20, 21]. Hence, the process of bubble nucleation is delayed in such regions. The effect of pre-existing baryon inhomogeneities on the quark-hadron phase transition depends on the profile of the inhomogeneities.

Assume for instance that the geometry is that of small regions with low baryon density in a background of higher density. Then, since the critical temperature is reached first in the low-density regions, the inhomogeneities may act as impurity sites where hadronphase bubbles can nucleate before the supercooling required for spontaneous nucleation is reached [13]. Thus, the QCD phase transition may proceed by inhomogeneous nucleation at $T_{c}$, rather than by supercooling and homogeneous nucleation with a rate $\Gamma$ per unit time and volume. Therefore, the size scale of the inhomogeneities determines the mean separation between centers of nucleation. This distance, in turn, sets the scale of the QCD inhomogeneities.

If, on the contrary, the inhomogeneities have the form of small lumps of high baryon density surrounded by a background of lower density, then the phase transition will be delayed inside the lumps. Since the Universe quickly reheats close to $T_{c}$, the high density regions stay in the quark-gluon plasma phase, while the hadron-phase bubbles grow outside. This also affects the QCD generation of inhomogeneities, since baryon number tends to accumulate in the deconfined quark phase, as explained above. As a consequence, inhomogeneities generated at the electroweak scale may be amplified at the QCD phase transition, and therefore indirectly affect primordial nucleosynthesis [16].

In this paper we study the formation of baryon density inhomogeneities in a baryongenerating electroweak phase transition. This issue has been previously investigated by Heckler [3] for the minimal standard model. However, as we already mentioned, electroweak baryogenesis is possible only in extensions of the SM. The function $n_{B}\left(v_{w}\right)$, as well as the dynamics of the phase transition, can be quite different in such extensions. As a consequence, the kind of inhomogeneities that form may be quantitatively as well as qualitatively different. In particular, in Ref. [3] the wall velocity was supposed to lie well on the right of $v_{\text {neak }}$, and a dependance $n_{B} \propto v_{w}^{-1}$ was assumed. In contrast, recent calculations [10, 11, 22] show that the initial velocity is likely to be around $v_{\text {peak }}$, at least in the case of the minimal supersymmetric standard model (MSSM). In such a case, the velocity slow-down may occur completely on the left of the peak [14] and, as we shall see, yield the opposite behavior, $n_{B} \propto v_{w}$.

In sec. II we analyze the dependance of $n_{B}$ on $v_{w}$ in the whole range of (non-relativistic) wall velocities. In sec. III we study the evolution of the wall velocity using analytic approximations which allow to determine the parametric dependance of the amplitude and shape of the inhomogeneities. Unfortunately, such approximations give only a qualitative picture, so we also make a more numerical investigation of the electroweak phase transition: In section IV] we derive appropriate equations for the evolution of temperature and fraction of volume occupied by the broken-symmetry phase. We integrate this set of equations numerically and 
calculate the baryon number density. In section $\nabla$ we present the results of this calculation. We consider different values of the parameters, which include the case of the MSSM. Finally, in section VI] we discuss the possibility that the electroweak baryon inhomogeneities have an effect on the QCD phase transition. We argue that this is possible in some extensions of the SM. Our conclusions are summarized in section VII

\section{DEPENDANCE OF ELECTROWEAK BARYOGENESIS ON THE WALL VELOCITY}

The baryon asymmetry originates from the chiral quark asymmetry in the symmetric phase that is generated as a consequence of the CP violating currents coming from the wall. The density of left-handed particles in front of the bubble walls can be calculated by considering a set of coupled diffusion equations of the form (see e.g. [23, 24])

$$
\dot{n}_{i}=D_{i} \nabla^{2} n_{i}-\sum \Delta_{a i} \Gamma_{a} \frac{\Delta_{a j} n_{j}}{k_{j}}+\gamma_{i},
$$

for all particle species $i$ that have $C P$ violating interactions with the bubble wall. Here, $D_{i}$ are the diffusion constants, $\Gamma_{a}$ are the rates of reactions that change the number of particle $i$ by an amount $\Delta_{a i}$ (the corresponding rates per unit volume are $T^{3} \Gamma_{a} / 6$ ), $k_{i}$ are statistical factors: $k_{i}=g_{i}$ for fermions, $k_{i}=2 g_{i}$ for bosons, where $g_{i}$ is the number of degrees of freedom of species $i$, and $\gamma_{i}$ are the rates of generation of axial numbers at the wall, which depend on the Higgs profile.

In general, the diffusion equations (2) must be solved numerically. However, several approximations can be made in order to simplify the problem [10, 11, 24, 25]. For instance, the diffusion constants for all quarks can be assumed to be nearly the same, and some of the rates $\Gamma_{a}$ (e.g., those corresponding to strong sphalerons and trilinear Higgs-quark interactions) can be assumed to be fast enough to be in thermal equilibrium. As a consequence, several of the particle densities can be eliminated algebraically, leaving only a few equations to deal with. In order to avoid entering into the details of any specific model, we will assume that all the $n_{i}$ can be written in terms of just one of them, so only one diffusion equation needs to be solved (see e.g. [24]). This will allow us to find an analytical solution for the baryon density, which can be easily inserted in the numerical calculation of the evolution of the phase transition.

Therefore, we can write a diffusion equation for the density of left-handed fermions with effective values of $D, \Gamma$, and $\gamma$,

$$
D n_{L}^{\prime \prime}+v_{w} n_{L}^{\prime}-\Gamma n_{L}+\gamma=0
$$

where we have made the usual assumption of a planar wall which moves non-relativistically to the right, so $\gamma_{i}$ and $n_{i}$ depend only on $z-v_{w} t$. The diffusion constant $D$ for the chiral asymmetry depends on the particle spectrum of the specific model. In supersymmetric extensions of the SM, the main contributions to $D$ come from the Higgs sector and give $D \sim 100 T^{-1}$ [24]. The effective axial quark number relaxation rate $\Gamma$ receives contributions from the helicity flip due to the top quark mass and from Higgs self-interactions. In the wall frame, it can be approximated by $[10,11,24] \Gamma(z)=\tilde{\Gamma} \theta(-z)$, where $\theta$ is Heaviside's function and $\tilde{\Gamma} \sim 10^{-1} T$. The weak sphaleron processes are very slow, so they can be disregarded for relatively large wall velocities. However, we will consider a range of velocities that may 
include very small values of $v_{w}$. Therefore, we should take into account that the effective rate $\Gamma$ in the symmetric phase is not zero but of the order of the weak sphaleron rate. Consequently, we will assume that $\Gamma=\tilde{\Gamma}$ in the broken-symmetry phase, and $\Gamma=a \Gamma_{w s}$ in the symmetric phase, where $a$ is a numerical constant that depends on the degrees of freedom contributing to Eq. (3), and $\Gamma_{w s} \sim 10^{-6} T[25]$.

Thus, Eq. (3) splits into two identical equations for $z<0$ and $z>0$. The solution is of the form

$$
\begin{aligned}
n_{L} & =A e^{-\omega_{+} z}+B e^{-\omega_{-} z} \\
& -\left[D\left(\omega_{+}-\omega_{-}\right)\right]^{-1} \int_{0}^{z}\left[e^{-\omega_{-}\left(z-z^{\prime}\right)}-e^{-\omega_{+}\left(z-z^{\prime}\right)}\right] \gamma\left(z^{\prime}\right) d z^{\prime}
\end{aligned}
$$

where $\omega_{ \pm}, A$ and $B$ are different on each side of the wall. The parameters $\omega_{ \pm}$are given by

$$
\omega_{ \pm}=v_{w} / 2 D \pm \sqrt{\left(v_{w} / 2 D\right)^{2}+\Gamma / D}
$$

and the constants $A$ and $B$ are determined by the boundary conditions $n_{L}( \pm \infty)=0$ and continuity of $n_{L}$ and $n_{L}^{\prime}$ at $z=0$. Their general expressions are rather cumbersome and can be found in the appendix. If the $C P$ violating source $\gamma$ is localized on the wall, then outside the wall $n_{L}(z)$ has a simple exponential dependence [notice that, since $\omega_{+}>0$ and $\omega_{-}<0$, only one exponential function survives on each side of the wall in Eq. (41)].

Since baryon number violation takes place in the symmetric phase, we are only interested in the solution for $z>0$,

$$
n_{L}(z)=A e^{-\omega_{s+} z},
$$

where $\omega_{s+}$ is given by Eq. (15), with $\Gamma=a \Gamma_{w s}$, and the constant $A$ is an integral of $\gamma(z)$ times a combination of exponentials. The source $\gamma$ is proportional to $v_{w}$, since it is given by the flux of particles that reflect from the moving wall [26]. Thus, $A$ is the product of $v_{w}$ times a factor that depends on the details of $C P$ violation at the bubble wall. Although this factor can be velocity-dependent, in general it is not very sensitive to $v_{w}$. In the appendix we check this assertion using an approximation for the source $\gamma(z)$. According to these considerations, we will assume that $A \propto v_{w}$. Since we are interested in density contrasts caused by a variation of the wall velocity, we only need to determine the baryon density $n_{B}$ up to a constant factor independent of $v_{w}$ (we assume that the $C P$ violation is enough to generate the observed BAU).

The baryon number density also satisfies a diffusion equation, similar to Eq. (3). In this case, only sphaleron processes are relevant, so we have

$$
D_{q} n_{B}^{\prime \prime}+v_{w} n_{B}^{\prime}-3 \Gamma_{w s} \theta(z)\left(n_{L}(z)+b n_{B}\right)=0 .
$$

Here, $D_{q} \sim 6 T^{-1}$ is the diffusion coefficient for quarks, and the numerical factor $b$ depends on the particle spectrum. The term proportional to $n_{B}$ accounts for baryon number relaxation when the wall velocity is small [10, 11]. The chiral density $n_{L}$ acts as a source term in Eq. (17) and is given by Eq. (6) .

The solution to Eq. (17) for $z \leq 0$ is a constant, $n_{B} \equiv n_{B}(0)$. For $z>0, n_{B}$ has the form of Eq. (4), with $\gamma(z)=3 \Gamma_{w s} n_{L}(z)$ and

$$
\omega_{B \pm}=v_{w} / 2 D_{q} \pm \sqrt{\left(v_{w} / 2 D_{q}\right)^{2}+3 b \Gamma_{w s} / D_{q}}
$$


The conditions of continuity at $z=0$ and vanishing baryon number at $z=+\infty$ (i.e., far in the symmetric phase) give the value of the constant baryon density in the broken symmetry phase,

$$
n_{B}=\frac{3 \Gamma_{w s}}{D_{q} \omega_{B+}} \int_{0}^{\infty} n_{L}(z) e^{\omega_{B-} z} d z .
$$

The parameter $\omega_{B-}$ is often approximated by $\omega_{B-} \simeq-3 b \Gamma_{w s} / v_{w}$, which is correct for $v_{w}^{2} \gg$ $4 D_{q} \Gamma_{w s}$, i.e., for $v_{w} \gtrsim 10^{-2}$. Eq. (9) can be easily integrated using Eq. (6),

$$
n_{B}=\frac{C v_{w}}{\left(v_{w}+\sqrt{v_{w}^{2}+4 D_{q} 3 b \Gamma_{w s}}\right)\left(v_{w}+\sqrt{v_{w}^{2}+4 D a \Gamma_{w s}}\right)+3 b D \Gamma_{w s}},
$$

where $C$ is a constant.

The analytic approximation (10) for the dependance of the BAU on the wall velocity is in reasonable agreement with other approximations and numerical calculations [10, 11, 14, 24]. Two quantitative differences with some works arise due to our inclusion in Eq. (3) of a non-vanishing rate $\Gamma \sim \Gamma_{w s}$ in the symmetric phase, and the inclusion of the diffusion coefficient for quarks in Eq. (17). In Fig. 11 we plot $n_{B}\left(v_{w}\right)$ for $a=3 b=1$. We see that the baryon density has a peak, and tends to zero for small or large wall velocities ${ }^{1}$. For $D_{q} \ll D$, the peak is close to $v_{w}=\sqrt{D \Gamma_{w s}}$. This can be seen either numerically or by making approximations of Eq. (10) for the different possible ranges of $v_{w}$. Furthermore, it can be easily seen that Eq. (10) has the expected behavior on each side of the peak, namely, $n_{B} \propto v_{w}$ for $v_{w} \ll \sqrt{D \Gamma_{w s}}$, and $n_{B} \propto v_{w}^{-1}$ for $v_{w} \gg \sqrt{D \Gamma_{w s}}$.

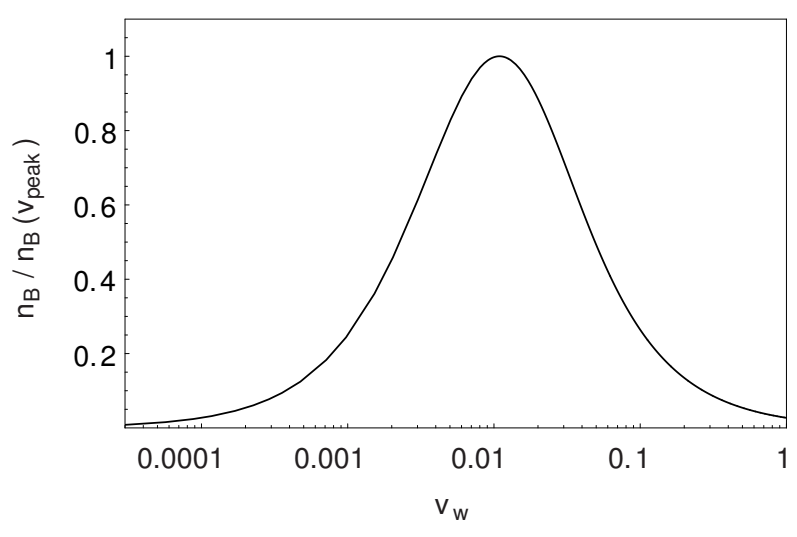

FIG. 1: Baryon number density as a function of the wall velocity

As we shall see, the wall velocity decreases during the phase transition as a consequence of latent heat release. Therefore, inside a given bubble, the local value of the baryon number density at a distance $r$ from the center of nucleation is given by $n_{B}(r)=n_{B}\left[v_{w}(t)\right]$, where $t$ is the moment at which the bubble wall has passed through $r$, i.e., $t$ is given by $R(t)=r$. The total BAU is the volume average $B=\int n_{B}\left[v_{w}(t)\right] d f$, where $f(t)$ is the fraction of volume occupied by bubbles. We remark that in this work we are assuming that $B$ gives the observed baryon abundance, which depends on the value of the constant $C$ in Eq. (10). We are interested in the relative variation of the baryon number density left behind by the

\footnotetext{
${ }^{1}$ Notice however that this approximation breaks down for $v_{w}$ close to 1.
} 
bubble walls, which does not depend on this constant, and can be computed from Eq. (10) once the time dependance of the wall velocity is known. Still, before closing this section we would like to comment on the effects of the velocity decrease on the total amount of baryons $B$.

If the initial velocity is $v_{i} \gg v_{\text {peak }} \sim \sqrt{D \Gamma_{w s}}$, a decrease of $v_{w}$ produces an enhancement of the BAU [3]. On the contrary, for $v_{i} \ll v_{\text {peak }}$, a velocity decrease will cause a suppression of the generated baryon asymmetry [14]. It is interesting to notice that in the case of an enhancement the effect can be quite large; however, in the case of a suppression the electroweak baryogenesis scenario will not be harmed significantly. Indeed, the volume spanned by a bubble wall while the velocity is closer to the initial value $v_{i}$ is in general of the same order of that spanned with $v_{w}$ closer to the minimum velocity $v_{m}$. Assuming that $n_{B}$ takes very different values $n_{B}\left(v_{i}\right)$ and $n_{B}\left(v_{m}\right)$ in each of these volumes, then the total baryon number density will be of the order of the largest one. Even in models in which baryon densities with opposite sign are formed in different regions, the suppression to the total BAU should be of order one. Otherwise it would require a fine tuning between the dynamics of the phase transition and the baryogenesis mechanism.

\section{PHASE TRANSITION AND BARYON INHOMOGENEITIES}

The electroweak phase transition takes place in the radiation-dominated era, at temperature $T_{c} \sim 100 \mathrm{GeV}$, when the expansion rate is $H_{c}=1 / 2 t_{c}$ and the age of the Universe is given by

$$
t_{c}=\xi M_{p} / T_{c}^{2} .
$$

Here, $M_{p}$ is the Planck Mass, and the parameter $\xi$ is related to the light degrees of freedom of the plasma, $g_{*}$, by $\xi=\sqrt{90 / 32 \pi^{3} g_{*}}$ (at the electroweak scale, $g_{*} \sim 100$ and $\xi \simeq 1 / 34$ ). The characteristics of the transition are determined by the free energy density difference $V(\phi, T)$ between a value $\phi \neq 0$ of the Higgs field VEV (corresponding to the brokensymmetry phase) and $\phi=0$ (symmetric phase). In a first-order phase transition, $V(\phi, T)$ has a non-zero minimum $\phi_{m}(T)$ coexisting with the minimum $\phi=0$ and separated from it by a barrier. Then, the quantity $V(T) \equiv V\left(\phi_{m}(T), T\right)$ gives the pressure difference between the two phases. In the simplest case, four parameters characterize the behavior of $V(\phi, T)$, namely, the critical temperature $T_{c}$ at which $V\left(T_{c}\right)=0$, the value of the order parameter at $T_{c}, \phi_{m}\left(T_{c}\right) / T_{c}$, the latent heat (i.e., the energy density discontinuity at $T=T_{c}$ ), given by

$$
L=T_{c}(d V / d T)_{T_{c}}
$$

and the tension of the bubble wall,

$$
\sigma=\int\left(\frac{d \phi}{d r}\right)^{2} d r
$$

where $\phi(r)$ is the wall profile at $T=T_{c}$.

An important parameter, which is not directly related to the free energy but is relevant for the dynamics of the phase transition is the friction coefficient $\eta$. In a first-order phase transition the system supercools, so bubbles nucleate at a temperature $T_{N}<T_{c}$. At $T \leq T_{N}$ bubbles expand with a velocity that is determined by the pressure difference between the 
broken-symmetry phase inside the bubbles and the symmetric phase that surrounds them, and by the friction of the wall with the plasma [8, 22, 27, 28],

$$
v_{w}(T)=-V(T) / \eta
$$

The electroweak wall velocity is subsonic, i.e. $v_{w}<c_{s}$, where $c_{s}=1 / \sqrt{3}$ is the velocity of sound in the relativistic plasma. Therefore the bubble expands as a deflagration. The deflagration front is the bubble wall, where the value of $\phi$ changes from 0 to $\phi_{m}$ and the energy (12) is released. A shock wave is formed in front of the wall, which is preceded by a supersonic shock front [29]. The energy released in the deflagration process is transmitted to the fluid and distributed between the two fronts. Most of it is closer to the phase transition front than to the shock front [3, 29], so a temperature profile arises. However, if $v_{w} \ll c_{s}$ (which is the case of the electroweak bubbles), part of this heat is carried away far in front of the wall and can influence other bubbles. If $v_{w}$ is small enough, one can assume that the released latent heat has enough time to distribute uniformly and equilibrate the temperature everywhere [3]. We will thus assume for simplicity that the only effect of the shock wave is to cause a uniform reheating of the plasma. This will be a good approximation if the time it takes the latent heat to get distributed throughout space (which depends on the bubble separation) is much shorter than the time scales involved in the development of the phase transition. We will examine the consistency of this approximation in section V]

The phase transition thus develops essentially in two steps (see e.g. [13]). The first stage is characterized by a quick increase of temperature and a decrease of $v_{w}$, as the latent heat reheats the plasma. After a certain time $\delta t_{1}$ the plasma has reheated to a temperature that is close to the critical one, and the system enters a phase-equilibrium or slow-combustion stage [1, 30]. During this second stage the latent heat release is compensated by the expansion of the Universe, and the temperature remains almost constant. Since $T \simeq T_{c}, V(T) \simeq 0$, so bubble expansion slows down significantly, and bubble nucleation effectively stops. Hence, this stage lasts for a longer time $\delta t_{2}$.

The time $\delta t_{\Gamma}$ during which bubbles nucleate is much less than $\delta t_{1}$ and $\delta t_{2}$ [13]. This is due to the quick variation of the nucleation rate $\Gamma(T)$ with temperature. Therefore, it is a good approximation to assume that all the bubbles nucleate at the beginning of the phase transition (this is confirmed by our numerical calculation). Hence, the number of bubbles remains fixed during the phase transition, and their number density can only change due to the dilution caused by the expansion of the Universe. However, the time scale of the electroweak phase transition is much less than the age of the Universe, so this effect is negligible. This means that when the phase transition completes, all the bubbles have roughly the same size, which is $\sim n_{b}\left(T_{N}\right)^{-1 / 3}$.

The size scale of the inhomogeneities is given by the size of the bubbles, and hence by the distance between centers of nucleation. Their shape depends on the distances travelled by the bubble walls during the times $\delta t_{1}$ and $\delta t_{2}$, and on the baryon-number density generated during each stage. The amplitude of the inhomogeneities (i.e., the contrast between the highest and the lowest baryon number densities) is determined by the value $v_{i}$ of the wall velocity at $T \simeq T_{N}$ and by its minimum value $v_{m}$ during the phase equilibrium stage.

Obtaining quantitative estimates of the phase transition from analytical approximations is a difficult task. On one hand, the dynamics involves integro-differential equations which complicate the analysis. On the other hand, the exponential dependance of the nucleation rate with temperature introduces a large amount of uncertainty in the estimations. Nevertheless, an analytical inspection will provide a qualitative picture of the inhomogeneities 
and will be useful to study their parametric dependance. We dedicate the rest of this section to such a study. For that, we use the analytical expressions derived in Ref. [13]. In the next sections we will check these results by numerically integrating the equations for the progress of the phase transition.

\section{A. Dynamics of the electroweak phase transition}

Bubbles begin to nucleate immediately after $T$ becomes smaller than $T_{c}$. However, for $T$ close to $T_{c}$ the nucleation rate is vanishingly small, so the number of bubbles is insignificant. The onset of nucleation can be defined as the moment at which there are enough bubbles so that they begin to feel the presence of each other. More precisely, we define the temperature $T_{N}$ as that at which the mean separation between bubbles equals the distance travelled by sound waves (which carry latent heat) since time $t_{c}$ [13]. Shortly after that, the temperature reaches its minimum value $T_{m}$, say at time $t_{m}$, and then increases due to the release of latent heat. Since the nucleation rate $\Gamma(T)$ reaches its maximum at the minimum temperature $T_{m}$, and is extremely sensitive to the temperature ${ }^{2}$, it is sharply peaked at $t=t_{m}$.

If the temperature $T_{N}$ is close enough to $T_{c}$, the thin wall approximation can be used to estimate the nucleation rate. In this case, the number density of bubbles is given by 13 .

$$
n_{b} \sim \frac{2 \pi \sigma^{6} \eta^{3} T_{c}^{2}}{9 \xi^{5} L^{8}}\left(\frac{T_{c}}{M_{P}}\right)^{3}\left(\frac{T_{c}}{T_{c}-T_{m}}\right)^{9} T_{c}^{3},
$$

The difference $T_{c}-T_{m}$ can be roughly approximated by $T_{c}-T_{N}$, where $T_{N}$ is given by

$$
\left(\frac{T_{c}}{T_{c}-T_{N}}\right)^{2} \simeq \frac{3 L^{2} T_{c}}{16 \pi \sigma^{3}} K
$$

and

$$
K=4 \log \left(2 \xi M_{p} / T_{c}\right)+\log \left(3 L^{2} T_{c} / 8 \pi \sigma^{3}\right)+6 \log \left[\left(T_{c}-T_{N}\right) / T_{c}\right]
$$

is a dynamical factor related to bubble nucleation at $T \simeq T_{N}$. This factor depends on several parameters but its value is dominated by the first term, so $K \sim 100$.

Notice that the number of bubbles depends on the friction $\eta$, and thus on the wall velocity. This is because for large $v_{w}$ latent heat is released more quickly, so the plasma reheats faster and the nucleation rate turns-off sooner. The friction coefficient depends on the viscosity of the plasma and the profile of the bubble wall. In the case $\phi_{m}(T) \sim T$, which is required for baryogenesis, this dependence can be factorized in the form [13]

$$
\eta \simeq \tilde{\eta} T \sigma
$$

where $\tilde{\eta}$ is a dimensionless constant that depends only on the particle content of the plasma. The value of this parameter determines the initial velocity $v_{i}$. Using the approximation

\footnotetext{
${ }^{2}$ For $T$ close to $T_{c}$, the dependance of $\Gamma$ on $T$ is dominated by an exponential function of the form $\Gamma \sim \exp \left[-C /\left(T_{c}-T\right)^{2}\right]$.

${ }^{3}$ In this approximation we are missing a slight (logarithmic) dependance of $T_{c}-T_{m}$ on the friction coefficient, as can be deduced from Eq. (53) of Ref. [13].
} 
$V(T) \simeq V\left(T_{N}\right) \simeq L\left(T_{N}-T_{c}\right)$ in Eq. (14), we can write the initial wall velocity in the form

$$
v_{i} \simeq \frac{L\left(T_{c}-T_{N}\right)}{\eta T_{c}},
$$

which makes apparent a factor of $v_{i}^{-3}$ in Eq. (15).

As the Universe is reheated, bubble growth slows down. When bubbles have transferred enough energy to the plasma, the temperature reaches a value very close to $T_{c}$ and stops increasing. This happens if the latent heat $L$ is at least equal to the difference $\delta \rho=\rho\left(T_{c}\right)-$ $\rho\left(T_{N}\right)$, where

$$
\rho(T)=\pi^{2} g_{*} T^{4} / 30
$$

is the energy density of the plasma. If, on the contrary, $L<\delta \rho$, then the temperature does not get close to $T_{c}$ and the expansion does not slow-down significantly.

For $L>\delta \rho$, the critical temperature is reached when the fraction of volume occupied by bubbles is

$$
f_{1} \simeq \delta \rho / L
$$

The average bubble radius at the end of the reheating stage is thus

$$
R_{1} \simeq\left(\frac{f_{1}}{4 \pi n_{b} / 3}\right)^{1 / 3}
$$

Notice that in Eq. (22) the radius $R_{1}$ does not have a straight dependance on the value of the wall velocity during this stage, as one could expect. This is because a larger $v_{w}$ implies a shorter reheating time. In fact, the time $\delta t_{1}$ can be estimated as $\delta t_{1} \simeq R_{1} / v_{i}$. However, the bubble number density $n_{b}$ does depend on the initial value of $v_{w}$, so in the end $R_{1}$ does depend on $v_{i}$ and $\delta t_{1}$ does not.

After reheating, bubbles can release latent heat only at the rate at which this energy is taken away by the expansion of the Universe. Therefore, the rate of bubble expansion is given by the condition $L \dot{f} \simeq 4 \rho H$, where $H=\sqrt{8 \pi G \rho / 3}$ is the expansion rate (here, $G$ is Newton's constant). Since the fraction of volume in the broken-symmetry phase is $f(t) \simeq 4 \pi n_{b} R(t)^{3} / 3$, we can write $\dot{f} \simeq 4 \pi n_{b} R_{2}^{2} v_{m}$, where the mean radius $R_{2}$ during this stage is given by $n_{b} R_{2}^{3} \sim 1$. Therefore, the wall velocity during slow combustion is given by

$$
v_{m} \sim \frac{\rho H}{L n_{b}^{1 / 3}} .
$$

One could naively think that the velocities (19) and (23) are unrelated, since $v_{i}$ depends explicitly on the friction $\eta$, whereas $v_{m}$ is determined by the Hubble rate $H$. However, using the analytical approximations (15][17), the velocities in the two stages can be written as

$$
\begin{aligned}
v_{i} & \simeq K^{-1 / 2} \frac{1}{\eta}\left(\frac{16 \pi \sigma^{3}}{3 T}\right)^{1 / 2}, \\
v_{m} & \simeq 7 g_{*}^{2 / 3} K^{-3 / 2} \frac{\sigma^{5 / 2} T^{11 / 6}}{\eta L^{4 / 3}} .
\end{aligned}
$$

We see that in this approximation $v_{m}$ is also proportional to $\eta^{-1}$, so the ratio $v_{i} / v_{f}$ does not depend on $\eta$. This is a consequence of the fact that in Eq. (23), the number density 
of bubbles is proportional to $\eta^{3}$, whereas $\rho$ and $H$ are not directly related to $\eta$. Indeed, we have seen that $n_{b} \propto v_{i}^{-3}$, so $v_{m}$ is in fact proportional to $v_{i}$, the proportionality factor being determined by the dynamics of reheating. From Eqs. (24) we obtain

$$
\frac{v_{i}}{v_{m}} \simeq \frac{4}{7} \frac{\left(L / T^{4}\right)^{4 / 3}}{\sigma / T^{3}} \frac{K}{g_{*}^{2 / 3}} .
$$

Since $K$ and $g_{*}$ are both $\sim 100$, if $L$ and $\sigma$ were of order 1 in temperature units, then $v_{i} / v_{m} \lesssim 10$. However, in the case of the electroweak phase transition we have in general $L / T^{4} \lesssim 1$ and $\sigma / T^{3} \ll 1$, so $v_{i} / v_{m}$ can be quite large.

We remark that the initial velocity is determined by the viscosity of the plasma and does not depend on the dynamics of the phase transition, whereas the ratio $v_{i} / v_{m}$ depends only on the dynamics of the phase transition, so $v_{i}$ and $v_{i} / v_{m}$ are quite independent quantities.

A potentially important source of variation of $v_{w}$ is bubble coalescence. When bubbles occupy a fraction of volume $f \simeq 1 / 3$, they percolate. This means that at this point most bubbles are in contact, so they can group to minimize surface energy. This process contributes to the growth of bubbles and could dominate the wall velocity [1]. When $f \simeq 1 / 2$ coalescence stops because the regions of symmetric phase begin to form isolated bubbles surrounded by the broken-symmetry phase, so the interfaces are pushed again by the pressure difference between the two phases. Hence, there may be a different velocity variation for $1 / 3 \lesssim f \lesssim 1 / 2$. To ascertain whether this is the case we must compare the bubble growth rates caused by the two mechanisms. The growth rate due to coalescence is $\dot{f}_{c} \sim\left(\sigma n_{b} / \rho\right)^{1 / 2}$ 13. We compare it with the rate due to pressure difference during the slow combustion stage, $\dot{f}_{2} \sim \rho H / L$. Therefore we have

$$
\frac{\dot{f}_{c}}{\dot{f}_{2}} \sim \frac{L^{3 / 2}}{\sigma T^{3}} \frac{K^{3 / 2}}{g_{*}^{3 / 4} v_{i}^{3 / 2}}\left(\frac{T}{M_{p}}\right)^{1 / 2}<10^{-3} \frac{L^{3 / 2}}{\sigma T^{3}}
$$

for $v_{i} \gtrsim 10^{-2}$. For values of $L / T^{4} \sim 0.1-1$, it would be necessary that $\sigma / T^{3} \ll 10^{-3}$ for coalescence to dominate and produce a significant departure from the velocity behavior described above. This does not seem likely for typical values of the electroweak phase transition parameters, although in general $\sigma / T^{3} \ll 1.4$

\section{B. Generation of inhomogeneities}

The amplitude of the inhomogeneities is determined by the total variation of the wall velocity during the transition. It is clear that larger density contrasts will arise if the velocity variation occurs far from the baryogenesis peak in Fig. 1, i.e., in the region where the baryon asymmetry is most sensitive to $v_{w}$. If $v_{i}$ and $v_{m}$ are much larger than $v_{\text {peak }}$, we can use the approximation $n_{B} \propto v_{w}^{-1}$. If, on the contrary, $v_{i}, v_{m} \ll v_{\text {peak }}$, then the baryon density has a dependance $n_{B} \propto v_{w}$. In any of these two cases, the ratio of the baryon densities in the high- and low- density regions, $\epsilon=n_{B}^{\max } / n_{B}^{\min }$, is given by the ratio $v_{i} / v_{m}$.

\footnotetext{
${ }^{4}$ In section $\square$ we consider values of $\sigma / T^{3}$ in the range $10^{-3}-10^{-1}$. Notice that the values of $L$ and $\sigma$ are not unrelated since both depend on the parameters of the theory. As a consequence, lower values of $\sigma$ correspond in general to lower values of $L[13]$.
} 
In the intermediate case in which the variation of $v_{w}$ crosses the peak of $n_{B}, \epsilon$ may be much smaller.

In general the peak velocity is in the range $10^{-2} \lesssim v_{\text {peak }} \lesssim 10^{-1}[10,11]$. Hence, to give the largest possible amplitude, the wall velocity should be either $v_{w}<10^{-2}$ (in order to be $\ll 10^{-1}$ ) or $v_{w}>10^{-1}$ (so that $v_{w} \gg 10^{-2}$ ). In general, however, the electroweak bubble walls have an initial velocity $v_{i} \sim 10^{-1}-10^{-2}$ [22, 28], so the velocity variation will not occur too far from the peak of $n_{B}$. If the initial velocity is $\sim 10^{-1}$, then $v_{w}$ will most likely cross $v_{\text {peak }}$ during the transition. So, the most favorable situation is that in which $v_{i}$ is closer to $10^{-2}$. In this case the velocity will not be far from $v_{\text {peak }}$ initially, but will probably not traverse it either. Large inhomogeneities can then arise if the minimum velocity departs enough from $v_{i}$.

As we have seen, the velocity variation depends on $L$ and $\sigma$. According to Eq. (25), for large $L$ or small $\sigma$, the wall velocity will change considerably, and important baryon density contrasts may be formed. On the contrary, if $L$ is too small or $\sigma$ too large, the wall velocity will not change significantly during the transition. In this case, the baryon number density will be rather homogeneous. Notice that for $L \rightarrow 0$ there is no reheating at all, and we should have $v_{i} / v_{m} \rightarrow 1$, so Eq. (25) fails in this limit. The problem is that for very small $L$, the bubble expansion never enters the slow combustion stage, so the basic assumptions that lead to Eq. (23) for the minimum velocity break down.

Since all the bubbles nucleate in a short time $\delta t_{\Gamma}$ at the beginning of the transition, all the inhomogeneities should have the same size scale, determined by the separation between centers of nucleation, $d \sim n_{b}^{-1 / 3}$. As can be seen in Eqs. (15.18), $n_{b}$ is a very sensitive function of $L, \sigma$, and the temperature difference $T_{c}-T_{m}$, which in turn depends on $L$ and $\sigma$ too. Furthermore there is an additional dependance on $\sigma$ through the friction coefficient $\eta$. So, we cannot trust the dependance of $d$ on $L$ and $\sigma$ obtained from these approximations. On the other hand, we do not expect a significant dependance of $T_{m}$ on the friction, so we can rely on the behavior $n_{b} \propto v_{i}^{-3}$, which implies $d \propto v_{i}$.

The spherically symmetric inhomogeneities that originate inside the bubbles can have two basic geometries, depending on the relation between $v_{\text {peak }}, v_{i}$, and $v_{m}$. If $v_{i}<v_{\text {peak }}$, the baryon asymmetry generated at the bubble walls decreases with time as bubbles expand. Hence, a larger baryon number density is produced at the centers of bubbles, and then the geometry of the inhomogeneities is that of spheres of high baryon number density $\sim n_{B}\left(v_{i}\right)$, surrounded by regions with lower density $\sim n_{B}\left(v_{m}\right)$ (see Fig. 2). These spheres have radius $R_{1}$ and occupy a fraction of volume $f_{1}$, given by Eqs. (21) and (22).

In the case $v_{m}>v_{\text {peak }}$, the baryon production will be larger at the end of bubble expansion, and inhomogeneities will have the form of walls which surround spherical voids of radius $R_{1}$. Therefore, these walls have a width $w \sim\left(f_{1}^{-1 / 3}-1\right) R_{1}$ and occupy a volume fraction $f_{2}=1-f_{1}$. Of course, there is a third possibility, namely, that $v_{i}>v_{\text {peak }}$ and $v_{m}<v_{\text {peak }}$, in which case the structure of the inhomogeneities can be more complex. However, as explained above, we do not expect inhomogeneities with large amplitude when $v_{w}$ varies across $v_{\text {peak }}$, so this case is in fact irrelevant.

According to Eq. (21), the volume fraction $f_{1}$ is given by the ratio $\delta \rho / L$, which must be less than 1 in order to have acceptable reheating and velocity variation. By naturalness, $L$ is not likely to be too close to $\delta \rho$, so $f_{1}$ is not expected to be very close to 1 . This implies that the width $w$ should be at least of the same order of $R_{1}$. On the other hand, $f_{1}$ could be very small if $L \gg \delta \rho$. In this case, we would have inhomogeneities of a small size $R_{1} \sim\left(f_{1} / n_{b}\right)^{1 / 3}$, separated by wide walls of size $w \sim d \sim n_{b}^{-1 / 3}$. Notice however that $R_{1} \ll d$ is not likely. 

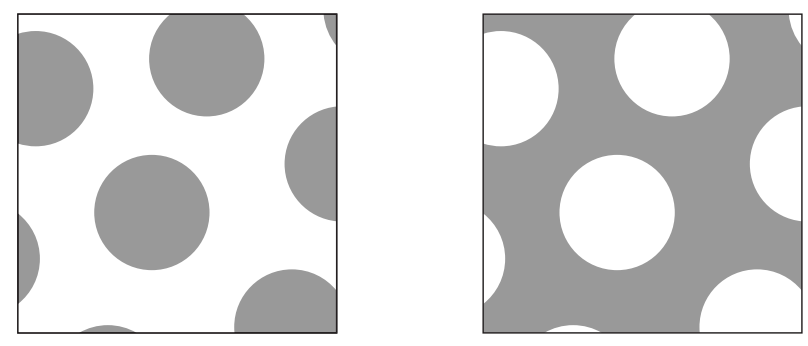

FIG. 2: Schematic picture of the geometry of the baryon inhomogeneities created during bubble expansion. Shaded regions correspond to high baryon number density. The left figure shows the case in which $v_{i}<v_{\text {peak }}$, whereas the right figure shows the case $v_{m}>v_{\text {peak }}$.

Indeed, a size $R_{1} \sim 0.1 d$ already requires a latent heat three orders of magnitude larger than the energy difference between $T_{N}$ and $T_{c}$. Although $\delta \rho / L$ can change significantly from one model to another [13], we do not expect that this ratio can be made arbitrarily small in a realistic theory, since both the latent heat and the amount of supercooling depend on the effective potential. As a consequence, we expect that the sizes of the spheres and walls in Fig. 2 will be similar for reasonable values of the parameters. This is important because if the size of the inhomogeneities is too small, they will be quickly erased by diffusion and will not survive until later epochs.

\section{NUMERICAL CALCULATION OF THE THE ELECTROWEAK PHASE TRANSITION}

In order to simplify our numerical calculation of the phase transition, we will use a free energy density of the form

$$
V(\phi, T)=D\left(T^{2}-T_{0}^{2}\right) \phi^{2}-E T \phi^{3}+\frac{\lambda}{4} \phi^{4} .
$$

It is well known that to one-loop order in perturbation theory and in the high-temperature approximation, the effective potential takes this form, where the parameters $D, T_{0}, E$, and $\lambda$ depend on the particle masses (see e.g. 31]). The actual form of the free energy depends on the model and differs in general from (27). This may happen, for instance, in the twoloop approximation, or in the case perturbation theory or the high-temperature expansion are not valid. In any case, this approximation is useful to simulate the phase transition in different models, which is convenient for a general treatment.

This is accomplished by adequately choosing the parameters in Eq. (27) so that the free energy gives the correct values for the thermodynamic parameters defined in the previous section. For instance, the critical temperature and latent heat are given by

$$
\begin{aligned}
T_{c} & =T_{0} / \sqrt{1-E^{2} / \lambda D}, \\
L & =8 D(E / \lambda)^{2} T_{c}^{2} T_{0}^{2}
\end{aligned}
$$

The first-order phase transition can take place between the temperatures $T_{c}$ and $T_{0}$, since in this temperature range the potential (27) has a local minimum at $\phi=0$, corresponding 
to the symmetric phase, and a global minimum at

$$
\phi_{m}(T)=\frac{3 E T}{2 \lambda}\left[1+\sqrt{1-\frac{8}{9} \frac{\lambda D}{E^{2}}\left(1-\frac{T_{0}^{2}}{T^{2}}\right)}\right],
$$

corresponding to the broken-symmetry phase. These two minima are separated by a barrier. At $T=T_{c}$ the minima are degenerate, and at $T=T_{0}$ the barrier disappears and the point $\phi=0$ becomes a maximum. The pressure difference between the two phases is $V(T) \equiv V\left(\phi_{m}(T), T\right)$. The order parameter of the phase transition is given by

$$
\phi_{c} / T_{c} \equiv \phi_{m}\left(T_{c}\right) / T_{c}=2 E / \lambda \text {. }
$$

The progress of the transition is characterized by the fraction of volume occupied by bubbles of the broken-symmetry phase. In the case of the electroweak phase transition, this is given by [27, 32, 33]

$$
f(t)=1-\exp \left\{-\frac{4 \pi}{3} \int_{t_{c}}^{t} \Gamma\left(T^{\prime}\right) R\left(t^{\prime}, t\right)^{3} d t^{\prime}\right\} .
$$

Here, $R\left(t^{\prime}, t\right)$ is the radius of a bubble that nucleated at time $t^{\prime}$ (and temperature $T^{\prime}$ ) and expanded until time $t$,

$$
R\left(t^{\prime}, t\right) \simeq \int_{t^{\prime}}^{t} v_{w}\left(T^{\prime \prime}\right) d t^{\prime \prime}
$$

where the wall velocity is given by Eq. (14), and we have neglected the initial radius of the critical bubble. The nucleation rate $\Gamma(T)$ is given by [34, 35]

$$
\Gamma \simeq T_{c}^{4} e^{-S_{3} / T}
$$

where $S_{3}(T)$ is the three-dimensional instanton action ${ }^{5}$, which coincides with the free energy of a critical bubble in unstable equilibrium between expansion and contraction,

$$
S_{3}=4 \pi \int_{0}^{\infty} r^{2} d r\left[\frac{1}{2}\left(\frac{d \phi}{d r}\right)^{2}+V(\phi(r), T)\right] .
$$

The configuration of the nucleated bubble can be obtained by extremizing this action. Hence it obeys the equation

$$
\frac{d^{2} \phi}{d r^{2}}+\frac{2}{r} \frac{d \phi}{d r}=\frac{\partial V}{\partial \phi}
$$

For $T \rightarrow T_{c}$ the radius of the bubble becomes infinite, so the profile of the wall can be easily calculated by neglecting the second term in (35). Then, we can readily compute the wall tension for the model (27),

$$
\sigma=\frac{2 \sqrt{2} E^{3}}{3 \lambda^{5 / 2}} T_{c}^{3}
$$

\footnotetext{
5 The prefactor of the exponential in (33) is roughly assumed to be of order $T^{4}$, since the rate is dominated by the exponential.
} 
This equation, together with Eqs. (28) and (30) determine completely the values of the parameters of $V(\phi, T)$ in terms of thermodynamical properties of the free energy.

Analytical approximations for the profile of the critical bubble introduce large errors in the nucleation rate. Therefore, we will use the numerical fit for $S_{3}$ given in Ref. [27],

$$
\begin{aligned}
\frac{S_{3}}{T} & =13.72 \frac{E}{\lambda^{3 / 2}} \alpha^{3 / 2} g(\alpha), \\
g(\alpha) & =1+\frac{\alpha}{4}\left(1+\frac{2.4}{1-\alpha}+\frac{0.26}{(1-\alpha)^{2}}\right),
\end{aligned}
$$

where $\alpha=\lambda D\left(T^{2}-T_{0}^{2}\right) / E^{2} T^{2}$. This dimensionless parameter can also be written as

$$
\alpha=\frac{T_{c}^{2}}{T_{0}^{2}} \frac{T^{2}-T_{0}^{2}}{T_{c}^{2}-T_{0}^{2}}
$$

where it is apparent that it varies from 1 to 0 as $T$ decreases from $T_{c}$ to $T_{0}$. [13]

Finally, the variation of temperature with time during the phase transition is given by

$$
T^{3}=\frac{T_{c}^{3} a_{c}^{3}}{a^{3}}+\frac{V^{\prime}(T)}{2 \pi^{2} g_{*} / 45} f .
$$

Here, the prime means total derivative with respect to temperature. The first term in $\mathrm{Eq}$ (39) gives the usual variation of $T$ in the adiabatic expansion, while the second term accounts for the release of entropy during the phase transition. The evolution of the scale factor $a(t)$ is given by the Friedman equation, $(\dot{a} / a)^{2} \equiv H^{2}=8 \pi G \rho(T) / 3$. Since the duration of the electroweak phase transition is much shorter than the age of the Universe, we can use the approximation $H \simeq H_{c}$ [13]. Note that in Eqs. (31) and (32) we have neglected the variation of length scales due to the expansion of the Universe. We cannot do the same in Eq. (39), since for $T \simeq T_{c}$ small changes of temperature are relevant. Indeed, the scale of temperature variation in a first order electroweak phase transition is given by $T_{c}-T_{0} \simeq\left(E^{2} / 2 \lambda D\right) T_{c} \ll T_{c}$.

\section{NUMERICAL RESULTS}

In order to solve numerically Eqs. (31,39) it is convenient to differentiate Eq. (39). At this stage we can make the approximations $a \simeq a_{c}, T \simeq T_{c}$, so we have

$$
\left(1-\frac{15}{2 \pi^{2} g_{*}} \frac{V^{\prime \prime}}{T^{2}} f\right) \frac{d T}{T_{c}}=-\frac{1}{2} \frac{d t}{t_{c}}+\frac{15}{2 \pi^{2} g_{*}} \frac{V^{\prime}}{T^{3}} d f .
$$

In addition, the variation of $f$ is given by

$$
d f=(1-f) 4 \pi v_{w}(t)\left[\int_{t_{c}}^{t} \Gamma R^{2} d t^{\prime}\right] d t
$$

Integration of these equations gives the values of the radius $R(t)$ and wall velocity $v_{w}(t)$ at every time. Then, the profile $n_{B}(r)$ is obtained with the aid of Eq. (10), as explained at the end of section [I]. For the numerical calculation we have made a simple discretization of Eqs. (40) and (41). We have used a time step much shorter than the duration of the 
phase transition $(\sim 1 / 1000)$, and we have verified that the result remained unchanged as this step decreased further. Therefore, the uncertainties of the numerical calculation are certainly smaller than the ones introduced by the analytical approximations used in the previous section.

\section{A. Parameter values}

Several extensions of the Standard Model provide a strong phase transition as well as enough CP violation for baryogenesis. Supersymmetric models were extensively studied in the literature, especially the case of the MSSM, which gives the required BAU in a light right-handed stop scenario [10, 11, 24, 36, 37]. Some alternatives to this model include non-minimal supersymmetric extensions (see e.g. [38]), the addition of heavy fermions to the SM [39], a dimension-six operator in the effective Higgs potential [40], or the presence of hypermagnetic fields during the phase transition [41, 42]. Therefore, we will consider various values of the parameters.

For the case of the MSSM, the thermodynamic parameters of the phase transition have been calculated [37]. Hence, we will take this case as a starting point for the parameter variation. We will consider different values of $L$ and $\sigma$, since these two parameters are the ones which most directly affect the dynamics of the phase transition. Thus, according to the non-perturbative study of Ref. [37], our reference values will be $T_{c} \simeq 85 \mathrm{GeV}, \phi_{c} / T_{c} \simeq 1$, $L / T_{c}^{4} \simeq 0.4, \sigma / T_{c}^{3}=0.01$. The viscosity of the plasma has also been studied for the MSSM in the light stop scenario [22]. We will consider values of the friction parameter $\tilde{\eta} \sim 0.1-10$, which give initial velocities ${ }^{6} v_{i} \sim 10^{-1}-10^{-2}$, in accordance with the results of Ref. [22].

To illustrate the kind of profiles of the baryon number density that can be created inside an expanding bubble, we will consider several values of the electroweak baryogenesis parameters. According to the discussions in section III the profile depends essentially on the position of $v_{\text {peak }}$ relative to the velocity variation. We have seen in section II that setting the values of the parameters $a$ and $b$ to $\sim 1$, the peak velocity is given by $v_{\text {peak }} \sim \sqrt{D \Gamma_{w s}}$. For the MSSM, $D \sim 100 T^{-1}$, so this gives the correct value $v_{\text {peak }} \sim 10^{-2}$. In other extensions of the SM the effective diffusion constant $D$ may differ from that value, and further deviations may arise through the precise values of $a$ and $b$. Therefore, we will consider values of $v_{\text {peak }}$ in the range $10^{-3}-10^{-1}$.

\section{B. Dynamics of the phase transition}

The evolution of the phase transition for these values of the parameters is shown in Fig. 3. We have plotted the variable $\alpha$ defined in Eq. (38), the fraction of volume $f$ occupied by bubbles, and the bubble wall velocity $v_{w}$, as functions of time. Notice that the evolution of the temperature and the fraction of volume is affected by the bubble wall friction only at the beginning of the phase transition. This is because during the reheating stage, the bubble expansion is dominated by friction. On the contrary, during the phase equilibrium stage the development of the phase transition is determined by the balance between the rate

\footnotetext{
${ }^{6}$ We obtained these relations between the values of $\tilde{\eta}$ and $v_{i}$ numerically. The values of $v_{i}$ obtained from Eqs. (24) and (18) are a factor $\simeq 2.5$ less than the numerical values.
} 
of latent heat release and that of cooling due to the expansion of the Universe, as explained in section III.

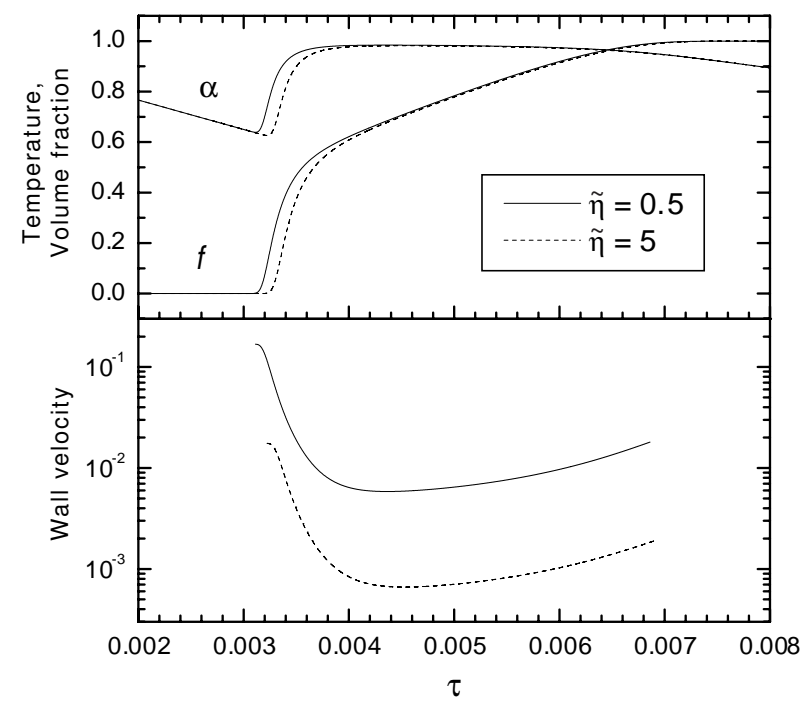

FIG. 3: Fraction of volume, temperature parameter $\alpha$, and wall velocity as functions of dimensionless time $\tau=\left(t-t_{c}\right) / t_{c}$.

We see that the wall velocity decreases by a factor that does not depend on the friction coefficient (and hence on the initial velocity $v_{i}$ ), as anticipated by the analytical approximations. In the present case the factor is $\simeq 30$, to be contrasted with the estimation (25), which for the parameters under consideration gives $v_{i} / v_{m} \simeq 100$. As expected, the discrepancy is of order 1.

Notice also that the wall velocity is not constant in the slow combustion stage, but it slowly begins to grow again after reaching its minimum value $v_{m}$. This happens because as the regions occupied by the symmetric phase become smaller, the energy must be released more quickly at the interfaces in order to compensate the cooling produced by the adiabatic expansion.

In the analysis of the inhomogeneities we have assumed that all bubbles begin to expand at the time $t_{i}$ at which the nucleation rate $\Gamma$ takes its maximum value. We have checked numerically that most of the bubbles nucleate at the very beginning, while the volume fraction increases from $10^{-3}$ to $10^{-2}$. Moreover, Fig. 4 shows that $\Gamma$ is active during a time interval $\delta t_{\Gamma}$ which is two orders of magnitude shorter than the total duration of the phase transition (compare with Fig. [3). This introduces a dispersion of about a $5 \%$ in the bubble radius at the end of the transition, which we define to be the moment $t_{f}$ at which $f=0.99$ (the initial and final times delimit the plot of the wall velocity in Fig. 31). Hence, there will not be inhomogeneities at different scales, although some dispersion in sizes will arise due to the fact that the centers of nucleation are randomly scattered throughout space.

\section{Profile of the baryon inhomogeneities}

In Figs. 5 and 6 we show the inhomogeneity profiles that arise for initial wall velocities slightly above 0.1 and slightly below 0.01 respectively, and different possible values of $v_{\text {peak }}$. 


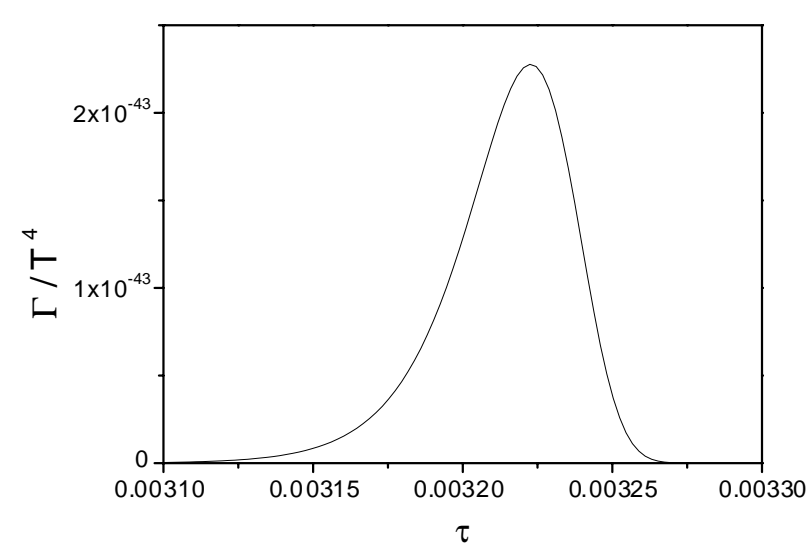

FIG. 4: The nucleation rate as a function of dimensionless time $\tau$ defined in Fig. 3.

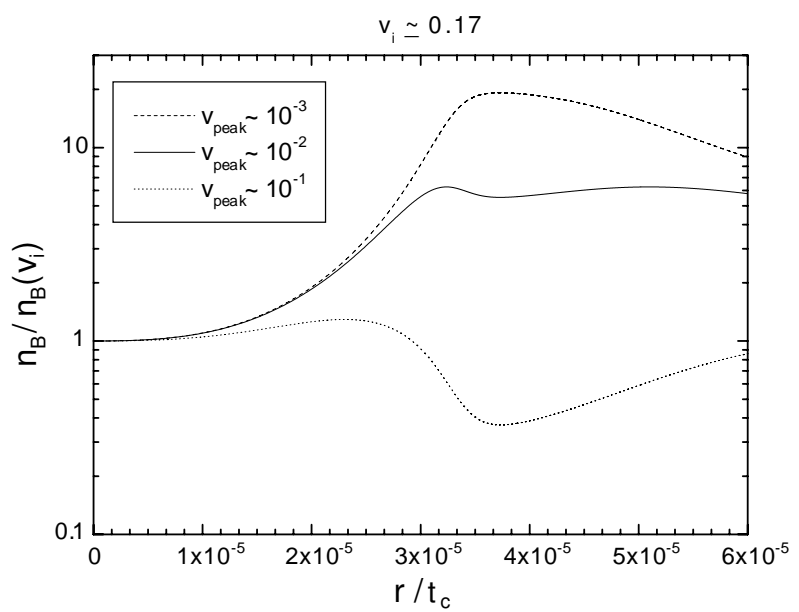

FIG. 5: Baryon number density as a function of the bubble radius $r$ in units of the age of the Universe $t_{c}$, for $v_{i} \sim 0.1$.

In the case $v_{i} \gtrsim 0.1$, we see that larger amplitudes are obtained for smaller values of $v_{\text {peak }}$, i.e., when $v_{i}$ is more distant from the baryon generation maximum. The profile for $v_{\text {peak }} \sim 10^{-3}$ in Fig. [5 is in good agreement with the result of Ref. [3], where a dependance $n_{B} \propto v_{w}^{-1}$ was assumed. The maximum of this curve corresponds to the minimum wall velocity $v_{m}$ reached during the phase transition, since in this case the velocity $v_{\text {peak }}$ is not attained. In the other curves, instead, the peak in $n_{B}\left(v_{w}\right)$ becomes manifest. In the case $v_{\text {peak }} \sim 10^{-2}$, the amplitude is smaller, and we observe the presence of two hills in the curve. This is because $v_{w}$ crosses $v_{\text {peak }}$ immediately before reaching the minimum $v_{m}$. Then it crosses the peak again due to the final acceleration. The case $v_{\text {peak }} \sim 10^{-1}$ gives still a smaller amplitude, since the velocity variation is more symmetric around the peak.

Fig. 6] shows the case $v_{i} \lesssim 10^{-2}$. As expected from the discussion of section III the profiles behave quite oppositely to the previous case. The largest amplitude is obtained for $v_{\text {peak }} \sim 10^{-1}$, and in this case the baryon number density is higher at the centers of the bubbles. The upper curve is similar to the lower curve of Fig. 5. because in this case the wall velocity crosses the peak. We see that when this happens, the amplitude is smaller and the baryon inhomogeneities are not clearly localized either in the interior or the exterior of 
the spheres of Fig 2

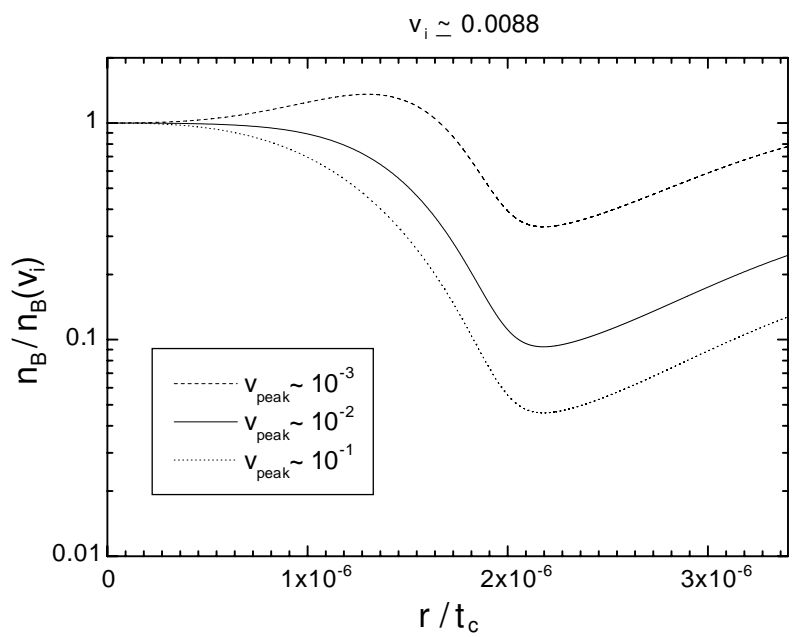

FIG. 6: Baryon number density as a function of the bubble radius $r$ in units of the age of the Universe $t_{c}$, for $v_{i} \sim 0.01$.

It is important to notice that these profiles describe the inhomogeneities produced by an average bubble that expands without colliding with other bubbles. Furthermore, bubble collisions may have some effect on the development of the phase transition. Since Eq. (31) takes into account overlapping of bubbles, the present simulation of the phase transition is in principle reliable in the whole time interval, with the possible exception of collisions and coalescence, which occur when the fraction of volume is $f \sim 0.3-0.5$. We have seen in section III that the dynamics of the electroweak phase transition is not affected by bubble coalescence. However, we are neglecting some possible effects, such as additional reheating due to bubble collisions. Such effects could introduce some distortion in the profiles shown in Figs. 5 and 6.

\section{Size and amplitude of the inhomogeneities}

The size scale of the inhomogeneities is given by the distance between centers of bubbles, $d \sim n_{b}^{-1 / 3}$ [3]. We plot this distance in Fig. [7 as a function of the bubble wall tension for different values of the latent heat and friction. Notice that, as expected, changing $\eta$ by an order of magnitude induces the same variation in $d$. We have studied the dependance of $d$ on the initial wall velocity in the range $10^{-3}<v_{i}<0.4$, and we have checked that the linearity $d \propto v_{i}$ is verified for the different values of $L$ and $\sigma$.

The maximum possible baryon density contrast $\epsilon$ is determined by the ratio $v_{i} / v_{m}$. In Fig. 8 we plot this ratio as a function of latent heat, for different values of $\sigma$. For MSSM parameters we find $v_{i} / v_{m} \sim 10-100$. We observe that for large $L$, Eq. (25) gives the correct order of magnitude and the exact parametric dependance. In contrast, for smaller values of latent heat, the behavior of the numerical curves changes abruptly. This was expected, since when $L \lesssim \delta \rho$ the reheating becomes insufficient and the phase transition ceases to have two well defined stages. Thus, the curves depart from the approximate behavior (25), and for $L \rightarrow 0, v_{m}$ approaches $v_{i}$, as required physically. 


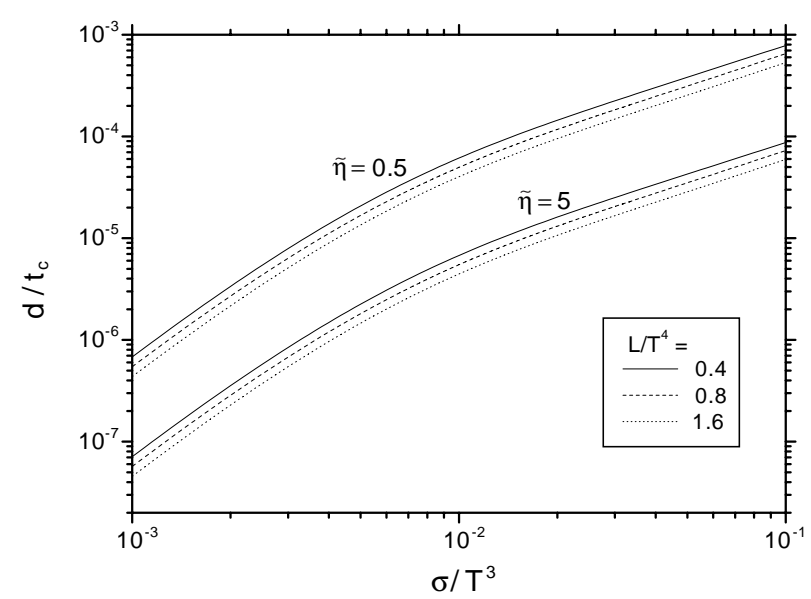

FIG. 7: Size of the inhomogeneities as a function of the bubble wall tension for different values of the latent heat and friction.

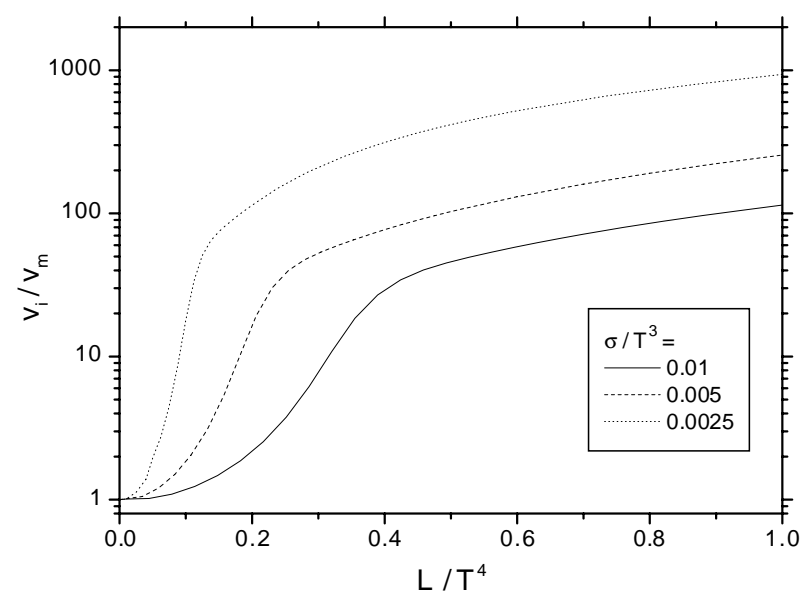

FIG. 8: The velocity ratio $v_{i} / v_{m}$ as a function of latent heat for different values of the bubble wall tension.

In this work we have assumed that the latent heat released as the bubbles expand is quickly distributed, so that the only effect is a global reheating. To estimate whether this approximation is correct or not, we must compare the time scales for the evolution of the phase transition with the time in which the latent heat liberated in a deflagration front reaches the neighboring bubbles. As seen in Fig. 3, for our reference values of the parameters the quick reheating stage occurs in a time $\delta t_{1} \sim 10^{-4} t_{c}$. On the other hand, the time it takes a shock front to travel the distance between bubbles is given by $\delta t_{s h} \sim d$. Hence, we expect that our treatment will be reliable for $d / t_{c} \ll 10^{-4}$. According to Fig. 7 , for $v_{i} \gtrsim 10^{-1}$ $(\tilde{\eta}=0.5)$ this definitely requires $\sigma / T^{3}<10^{-2}$. For smaller values of the initial wall velocity this condition is relaxed. After the system reheats, the transition proceeds more slowly. In this stage the time scale is $\delta t_{2} \sim 10^{-3} t_{c}$, and the approximation breaks down only for higher values of the wall velocity and tension, $v_{i} \sim 0.1, \sigma / T^{3} \sim 10^{-1}$. Since the amplitude of the inhomogeneities is larger for smaller values of $\sigma$ (see Fig. 8), in the interesting cases the global reheating approximation is valid. 


\section{EFFECT ON THE QUARK-HADRON PHASE TRANSITION}

The aim of this paper was to study the characteristics of the baryon inhomogeneities generated in the electroweak phase transition. However, we wish to comment briefly on the possibilities for these inhomogeneities to leave a sequel in the subsequent evolution of the Universe. The time scale for the wash-out of the inhomogeneities depends on their size and amplitude, and on the diffusive processes. For temperatures in the range $100 \mathrm{GeV}<\mathrm{T}<$ $1 \mathrm{MeV}$, the only important dissipation process is neutrino diffusion. A wide range of sizes and amplitudes is left almost unaffected by this process [17, 43]. Therefore, it is likely that electroweak inhomogeneities survive somewhat unchanged until the QCD scale [16, 17], in which case they may influence the dynamics of the quark-hadron phase transition. Below $1 \mathrm{MeV}$ other processes (namely, baryon and photon diffusion) become important and may completely erase the electroweak inhomogeneities before the nucleosynthesis epoch.

The evolution of baryon inhomogeneities between the epochs of $T=100 \mathrm{GeV}$ and $T=100 \mathrm{MeV}$ has been considered in Ref. [17]. According to this calculation, a baryon inhomogeneity which at the electroweak scale has amplitude $<10^{3}$ and size $>10^{-8} t_{c}$, corresponding to the cases considered in Figs. 7 and 8, may survive almost unchanged until the QCD epoch.

As shown in Fig. 8, in the MSSM inhomogeneities with amplitude $\epsilon_{\mathrm{MSSM}} \sim 10-100$ are likely. However, there exist several interesting scenarios for the electroweak phase transition 38, 39, 40, 41, 42] besides the MSSM. In some of them the phase transition is much stronger than in the latter. In such extensions of the SM, the parameters affecting the dynamics will differ significantly from those of the MSSM. In particular, larger values of the latent heat are expected in stronger phase transitions 13]. This leads in general to a larger $\epsilon$, as can be seen in Fig. 8. Further enhancement (or suppression) may also result from the variation of the wall tension. In fact, one expects larger values of $\sigma$ in stronger phase transitions, so in principle this effect goes in the opposite direction to that of latent heat. In any case, a sensible estimation of the inhomogeneity amplitude requires a calculation of $L$ and $\sigma$ in each particular case. Nevertheless, it is reasonable to expect that in some models amplitudes considerably larger than in the MSSM may arise. In such models we will possibly find $\epsilon \gg 100$.

As can be seen in Figs. [7 and 8, values of $L$ and $\sigma$ which give larger amplitudes, yield also smaller sizes. Such a correlation is important because inhomogeneities with larger amplitudes and smaller sizes are more easily diluted. Extrapolating our results beyond the ranges of parameters considered in the previous section, we see that for an amplitude $\sim 10^{4}$ we would have a size $d / t_{c} \sim 10^{-8}-10^{-7}$, which is still undamped by neutrino inflation. Larger amplitudes will probably be affected. For instance, according to the results of Ref. [17], an initial amplitude $\sim 10^{5}$ may decrease to $\sim 10^{4}$ if the size scale is $\lesssim 10^{-9} t_{c}$. Therefore, it seems unlikely that inhomogeneities generated in the electroweak phase transition may have an amplitude much larger than $10^{4}$ at the time of the QCD phase transition.

In Ref. 16], it was found that a significant effect on the latter is obtained for inhomogeneities with an amplitude $\epsilon \sim 10^{7}$. However, it was also noticed that smaller values of $\epsilon$ will have similar effects if the amount of supercooling is smaller than the one considered there, in accordance to Ref. [4]. Furthermore, in the treatment of Ref. 16] the possibility that the nucleation rate changes with the chemical potential (see e.g. 45]) was not considered. Taking into account this effect could affect strongly the discussion in Ref. [16], and therefore lead to a significantly different value of the inhomogeneity amplitude needed to 
affect this transition. Altogether, it is clear that the dynamics of the quark-hadron phase transition is still not fully understood, and we can sensibly expect that it may be affected by prior inhomogeneities with amplitudes of the order of those that are generated in the electroweak phase transition. We hope to address this issue in future work.

\section{CONCLUSIONS}

In this paper we have made a detailed study of the baryon number inhomogeneities that can be generated as a byproduct of electroweak baryogenesis. In particular, we have investigated analytically the parametric dependance of the inhomogeneities, and we have made a more precise calculation of the amplitude and size of the density contrasts by solving numerically the equations for the development of the electroweak phase transition. By doing that, we have checked several analytical approximations derived in Ref. [13] for the dynamics of the phase transition. An important difference between our treatment and previous analysis is that we have taken into account the fact that in general the baryon density has a maximum at a bubble wall velocity $v_{w}=v_{\text {peak }}$. We have accomplished this by deriving a simple analytical approximation for the dependance of the baryon asymmetry of the Universe on $v_{w}$.

The characteristics of the inhomogeneities depend on the parameters that determine the dynamics of the phase transition, namely, the latent heat $L$, bubble wall tension $\sigma$, and friction coefficient $\eta$, and on the parameters that govern the production of baryons, which are essentially the diffusion constant $D$ for the chiral quark asymmetry and the weak sphaleron rate $\Gamma_{w s}$. Some general features, however, are independent of these parameters. For instance, the spherical symmetry and the absence of a significant dispersion in sizes.

Indeed, we have shown that all the bubbles are nucleated in a short time interval at the beginning of the transition, and therefore have roughly the same radius. (We wish to remark here that this feature of first-order phase transitions may be important for other cosmological consequences as well, such as the formation of topological defects or magnetic fields. We will address these issues somewhere else.) Since the baryon inhomogeneities are generated in the walls of expanding bubbles and depend on the wall velocity, this implies that all inhomogeneities have approximately the same size, amplitude, and profile.

The size scale is given by the distance between centers of nucleation, so it depends only on the phase transition parameters $L, \sigma$, and $\eta$. In particular, we have seen that the higher the initial wall velocity, the larger the bubble separation, due to the sooner turn-off of the nucleation rate. The amplitude $\epsilon$ of the density contrasts may depend further on the baryogenesis parameters; more precisely, on $v_{\text {peak }} \simeq \sqrt{D \Gamma_{w s}}$. However, if $v_{w}$ stays far from $v_{\text {peak }}$ throughout the phase transition, $\epsilon$ is given by the ratio $v_{i} / v_{m}$ between the maximum and minimum values of $v_{w}$. We have seen that this ratio does not depend on the friction parameter, so in this case $\epsilon$ is only determined by $L$ and $\sigma$. If the wall velocity crosses the value $v_{\text {peak }}$ during the transition, then the amplitude is smaller than $v_{i} / v_{m}$.

The exact profile of the inhomogeneities, on the other hand, depends both on $v_{\text {peak }}$ and on the dynamics of the phase transition. In the case of the MSSM, the initial velocity is most likely close to the baryogenesis peak, $v_{i} \sim v_{\text {peak }} \sim 10^{-2}$ [11, 22]. As we have seen, this implies that the geometry is that of high-density spheres surrounded by low-density walls. This is contrary to the case of the SM, where wall-shaped inhomogeneities are formed [3], showing that both kinds of profile can arise in different models.

For MSSM values of the parameters $L$ and $\sigma$, we find amplitudes $\epsilon \lesssim 100$. Nevertheless, 
since the ratio $v_{i} / v_{m}$ grows with $L^{4 / 3}$ and with $\sigma^{-1}, \epsilon$ may be much larger in other extensions of the SM. We have seen that if this is the case, the electroweak baryon inhomogeneities will probably affect the dynamics of the quark-hadron phase transition, as discussed in Ref [16].

For the size of the inhomogeneities, we found in the case of the MSSM a value of order $10^{-6}-10^{-5}$ times the age of the Universe (in agreement with Ref. [3] ). However, this quantity is sensitive to $\eta$ and $\sigma$, and can deviate significantly from this value in other models.

These behaviors are illustrated in Figs. 7 and 8 . Our analytical expressions agree with the numerical computations within the order of magnitude. We have considered parameters that varied in a wide range in order to cover possible extensions of the SM, other than the MSSM. Furthermore, our results can be easily extrapolated beyond these ranges.

\section{Acknowledgements}

A. M. is grateful to M. Quirós for useful discussions, and to the Physics and Mathematics Institute at Michoacana University, where this work was initiated, for kind hospitality. The work by F. A. was supported by CIC-UMSNH and Conacyt grant 32399-E.

\section{APPENDIX A: THE CHIRAL ASYMMETRY}

In this appendix we find an analytical approximation for the density $n_{L}(z)$. In section 2 we have seen that this is of the form

$$
n_{L}=A e^{-\omega_{s+} z}
$$

and we argued that $A \propto v_{w}$, which is all we need to know for the present paper. However, this may not be true in general, so it is important to see under which conditions the coefficient $A$ depends only linearly on $v_{w}$.

As we have seen, the solution to Eq. (3) is given by Eqs. (4) and (5), with different values of $\Gamma$, and consequently of $\omega_{ \pm}, A$, and $B$ in the broken and symmetric phases. Specifically, $\Gamma_{b}=\tilde{\Gamma}, \Gamma_{s}=a \Gamma_{w s}$, where the index $b(s)$ stands for the broken (symmetric) phase. According to Eq. (5) we then have $\omega_{i \pm}=v_{w} / 2 D \pm\left[\left(v_{w} / 2 D\right)^{2}+\Gamma_{i} / D\right]^{1 / 2}$, and the constants $A_{i}$ and $B_{i}$ are given by the boundary conditions as explained in section [II. As a result, one obtains

$$
\begin{aligned}
& A_{b}=\left[D\left(\omega_{b+}-\omega_{b-}\right)\right]^{-1} \int_{-\infty}^{0} e^{\omega_{b+} z} \gamma(z) d z \\
& B_{s}=\left[D\left(\omega_{s+}-\omega_{s-}\right)\right]^{-1} \int_{0}^{\infty} e^{\omega_{s-} z} \gamma(z) d z
\end{aligned}
$$

and

$$
\begin{aligned}
& A_{s}=\frac{\omega_{b+}-\omega_{b-}}{\omega_{s+}-\omega_{b-}} A_{b}-\frac{\omega_{s-}-\omega_{b-}}{\omega_{s+}-\omega_{b-}} B_{s}, \\
& B_{b}=\frac{\omega_{b+}-\omega_{s+}}{\omega_{s+}-\omega_{b-}} A_{b}+\frac{\omega_{s+}-\omega_{s-}}{\omega_{s+}-\omega_{b-}} B_{s} .
\end{aligned}
$$

The source $\gamma$ is proportional to the wall velocity, $\gamma=v_{w} \tilde{\gamma}$. If $\tilde{\gamma}(z)$ is localized in some region around the wall, then it can be easily seen that the coefficients (A2) are such that 
outside this region only the exponential with the right sign of $\omega$ survives in each phase. To obtain an analytical result, we need to use an approximation for $\tilde{\gamma}$. The simplest approximation for a function $\tilde{\gamma}(z)$ that is localized inside the wall is a step function [24], say, $\tilde{\gamma}=\tilde{\gamma}_{0}$ for $-L_{w}<z<0$, and $\tilde{\gamma}=0$ outside. (The value of the constant $\tilde{\gamma}_{0}$ depends on the $C P$ violating force inside the wall.) Then, the solution for $z>0$ is of the form (A1), with

$$
A=\frac{\left(1-e^{-\omega_{b+} L_{w}}\right) v_{w} \tilde{\gamma}_{0}}{D \omega_{b+}\left(\omega_{s+}-\omega_{b-}\right)} .
$$

Notice that for $\tilde{\Gamma} \sim 10^{-1} T$, the relation $\left(v_{w} / 2 D\right)^{2} \ll \Gamma_{b} / D$ holds for any possible value of the wall velocity, as long as $D \gtrsim 10 T^{-1}$. So, the $\omega_{b \pm}$ are insensitive to the wall velocity, $\omega_{b \pm} \simeq \pm \sqrt{\tilde{\Gamma} / D}$. Furthermore, since $\Gamma_{s} \ll \Gamma_{b}$, we can also neglect $\omega_{s+}$ in Eq. (A4), and the coefficient $A$ becomes

$$
A \simeq v_{w} \frac{\tilde{\gamma}_{0}\left(1-e^{-L_{w} \sqrt{\tilde{\Gamma} / D}}\right)}{\tilde{\Gamma}},
$$

which is evidently linear in $v_{w}$. This expression for the coefficient $A$ coincides with the one obtained in Ref. [24]. On the other hand, the $z$-dependance in Eq. (A1) is different due to our inclusion of the sphaleron process in the diffusion equation for $n_{L}$. As we have seen, this modification does not introduce any qualitatively different behavior in the final result for the baryon number density. However, some quantitative $\mathcal{O}(1)$ difference in the BAU shows up for $v_{w}^{2} \lesssim D \Gamma_{w s}$, i.e., for $v_{w} \lesssim 10^{-2}$.

[1] E. Witten, Phys. Rev. D 30, 272 (1984).

[2] G. M. Fuller, G. J. Mathews and C. R. Alcock, Phys. Rev. D 37, 1380 (1988); J. H. Applegate and C. J. Hogan, Phys. Rev. D 31, 3037 (1985); H. Kurki-Suonio, Phys. Rev. D 37, 2104 (1988); J. Ignatius, K. Kajantie, H. Kurki-Suonio and M. Laine, Phys. Rev. D 50, 3738 (1994) arXiv:hep-ph/9405336.

[3] A. F. Heckler, Phys. Rev. D 51, 405 (1995) arXiv:astro-ph/9407064.

[4] R. H. Brandenberger, A. C. Davis and M. J. Rees, Phys. Lett. B 349, 329 (1995) arXiv:astro-ph/9501040.

[5] A. D. Sakharov, Pisma Zh. Eksp. Teor. Fiz. 5, 32 (1967) [JETP Lett. 5, 24 (1967 SOPUA,34,392-393.1991 UFNAA,161,61-64.1991)].

[6] A. G. Cohen, D. B. Kaplan and A. E. Nelson, Ann. Rev. Nucl. Part. Sci. 43, 27 (1993) arXiv:hep-ph/9302210; A. Riotto and M. Trodden, Ann. Rev. Nucl. Part. Sci. 49, 35 (1999) arXiv:hep-ph/9901362.

[7] M. E. Shaposhnikov, Nucl. Phys. B 287, 757 (1987); M. E. Shaposhnikov, Nucl. Phys. B 299, 797 (1988); A. I. Bochkarev and M. E. Shaposhnikov, Mod. Phys. Lett. A 2, 417 (1987);

A. I. Bochkarev, S. V. Kuzmin and M. E. Shaposhnikov, Phys. Rev. D 43, 369 (1991).

[8] B. H. Liu, L. D. McLerran and N. Turok, Phys. Rev. D 46, 2668 (1992); N. Turok, Phys. Rev. Lett. 68, 1803 (1992).

[9] A. E. Nelson, D. B. Kaplan and A. G. Cohen, Nucl. Phys. B 373, 453 (1992).

[10] J. M. Cline and K. Kainulainen, Phys. Rev. Lett. 85, 5519 (2000) arXiv:hep-ph/0002272; J. M. Cline, M. Joyce and K. Kainulainen, JHEP 0007, 018 (2000) arXiv:hep-ph/0006119; 
[11] M. Carena, J. M. Moreno, M. Quiros, M. Seco and C. E. Wagner, Nucl. Phys. B 599, 158 (2001) arXiv:hep-ph/0011055; M. Carena, M. Quiros, M. Seco and C. E. M. Wagner, Nucl. Phys. B 650, 24 (2003) arXiv:hep-ph/0208043.

[12] P. Y. Huet, K. Kajantie, R. G. Leigh, B. H. Liu and L. D. McLerran, Phys. Rev. D 48, 2477 (1993) arXiv:hep-ph/9212224; J. Ignatius, K. Kajantie, H. Kurki-Suonio and M. Laine, Phys. Rev. D 49, 3854 (1994) arXiv:astro-ph/9309059; H. Kurki-Suonio and M. Laine, Phys. Rev. Lett. 77, 3951 (1996) arXiv:hep-ph/9607382 ; A. Megevand, Int. J. Mod. Phys. D 9, 733 (2000) arXiv:hep-ph/0006177.

[13] A. Mégevand, Phys. Rev. D 69, 103521 (2004) arXiv:hep-ph/0312305.

[14] A. Mégevand, Phys. Rev. D 64, 027303 (2001) arXiv:hep-ph/0011019.

[15] J. H. Applegate, C. J. Hogan and R. J. Scherrer, Phys. Rev. D 35, 1151 (1987).

[16] S. Sanyal, Phys. Rev. D 67, 074009 (2003) arXiv:hep-ph/0211208.

[17] K. Jedamzik and G. M. Fuller, Astrophys. J. 423, 33 (1994) arXiv:astro-ph/9312063.

[18] M. B. Christiansen and J. Madsen, Phys. Rev. D 53, 5446 (1996) arXiv:astro-ph/9602071.

[19] Y. Hosotani, Phys. Rev. D 27, 789 (1983).

[20] M. A. Halasz, A. D. Jackson, R. E. Shrock, M. A. Stephanov and J. J. Verbaarschot, Phys. Rev. D 58, 096007 (1998) arXiv:hep-ph/9804290.

[21] Z. Fodor and S. D. Katz, Phys. Lett. B 534 (2002) 87 arXiv:hep-lat/0104001; JHEP 0203 (2002) 014 arXiv:hep-lat/0106002.

[22] P. John and M. G. Schmidt, Nucl. Phys. B 598, 291 (2001) [Erratum-ibid. B 648, 449 (2003)] arXiv:hep-ph/0002050.

[23] A. G. Cohen, D. B. Kaplan and A. E. Nelson, Phys. Lett. B 336, 41 (1994) arXiv:hep-ph/9406345.

[24] P. Huet and A. E. Nelson, Phys. Rev. D 53, 4578 (1996) arXiv:hep-ph/9506477.

[25] D. Bodeker, G. D. Moore and K. Rummukainen, Phys. Rev. D 61, 056003 (2000) arXiv:hep-ph/9907545; G. D. Moore and K. Rummukainen, Phys. Rev. D 61, 105008 (2000) arXiv:hep-ph/9906259; G. D. Moore, Phys. Rev. D 62, 085011 (2000) arXiv:hep-ph/0001216.

[26] A. G. Cohen, D. B. Kaplan and A. E. Nelson, Nucl. Phys. B 349, 727 (1991); M. Joyce, T. Prokopec and N. Turok, Phys. Rev. Lett. 75, 1695 (1995) [Erratum-ibid. 75, 3375 (1995)] arXiv:hep-ph/9408339; M. Joyce, T. Prokopec and N. Turok, Phys. Rev. D 53, 2958 (1996) arXiv:hep-ph/9410282.

[27] M. Dine, R. G. Leigh, P. Y. Huet, A. D. Linde and D. A. Linde, Phys. Rev. D 46, 550 (1992) arXiv:hep-ph/9203203.

[28] G. D. Moore and T. Prokopec, Phys. Rev. D 52, 7182 (1995) arXiv:hep-ph/9506475; Phys. Rev. Lett. 75, 777 (1995) arXiv:hep-ph/9503296;

[29] H. Kurki-Suonio, Nucl. Phys. B 255, 231 (1985).

[30] E. Suhonen, Phys. Lett. B 119, 81 (1982).

[31] M. Quiros, arXiv:hep-ph/9901312.

[32] A. H. Guth and E. J. Weinberg, Phys. Rev. D 23, 876 (1981).

[33] G. W. Anderson and L. J. Hall, Phys. Rev. D 45, 2685 (1992).

[34] I. Affleck, Phys. Rev. Lett. 46, 388 (1981).

[35] A. D. Linde, Nucl. Phys. B 216, 421 (1983) [Erratum-ibid. B 223, 544 (1983)]; Phys. Lett. B 100, 37 (1981).

[36] M. Carena, M. Quiros and C. E. M. Wagner, Phys. Lett. B 380, 81 (1996) arXiv:hep-ph/9603420; Nucl. Phys. B 524, 3 (1998) arXiv:hep-ph/9710401; B. de Car- 
los and J. R. Espinosa, Nucl. Phys. B 503, 24 (1997) arXiv:hep-ph/9703212; J. M. Cline and G. D. Moore, Phys. Rev. Lett. 81, 3315 (1998) arXiv:hep-ph/9806354; F. Csikor, Z. Fodor, P. Hegedus, A. Jakovac, S. D. Katz and A. Piroth, Phys. Rev. Lett. 85, 932 (2000) arXiv:hep-ph/0001087.

[37] M. Laine and K. Rummukainen, Nucl. Phys. B 597, 23 (2001) arXiv:hep-lat/0009025.

[38] M. Pietroni, Nucl. Phys. B 402, 27 (1993) arXiv:hep-ph/9207227]; A. Menon, D. E. Morrissey and C. E. M. Wagner, arXiv:hep-ph/0404184.

[39] M. Carena, A. Megevand, M. Quiros and C. E. M. Wagner, arXiv:hep-ph/0410352.

[40] C. Grojean, G. Servant and J. D. Wells, arXiv:hep-ph/0407019.

[41] P. Elmfors, K. Enqvist and K. Kainulainen, Phys. Lett. B 440, 269 (1998) arXiv:hep-ph/9806403 ; K. Kajantie, M. Laine, J. Peisa, K. Rummukainen and M. E. Shaposhnikov, Nucl. Phys. B 544, 357 (1999) arXiv:hep-lat/9809004.

[42] A. Ayala, J. Besprosvany, G. Pallares and G. Piccinelli, Phys. Rev. D 64, 123529 (2001) arXiv:hep-ph/0107072; A. Ayala, G. Piccinelli and G. Pallares, Phys. Rev. D 66, 103503 (2002) arXiv:hep-ph/0208046.

[43] A. Heckler and C. J. Hogan, Phys. Rev. D 47, 4256 (1993).

[44] B. Banerjee and R. V. Gavai, Phys. Lett. B 293, 157 (1992) arXiv:astro-ph/9205006.

[45] L. P. Csernai and J. I. Kapusta, Phys. Rev. D 46, 1379 (1992). 